\title{
Pattern of The Buccal Branch of Facial Nerve Among A Sample of Sudanese Subjects: A Multicenters Study
}

\author{
Yousif I Eltohami ${ }^{1 *}$, Shiang Fu Huang ${ }^{2}$ and Ahmed M Suleiman ${ }^{3}$ \\ ${ }^{1}$ Assistant professor of Oral and Maxillofacial Surgery, University of Khartoum, Sudan \\ ${ }^{2}$ Professor of Otolarynggology, head and neck surgery, Chang Gung Memorial Hospital, Taiwan \\ ${ }^{3}$ Professor of Oral \& Maxillofacial Surgery, University of Khartoum, Sudan
}

*Corresponding author: Yousif I Eltohami, Assistant professor of Oral and Maxillofacial Surgery, University of Khartoum, Sudan.

Received Date: August 14, 2019

Published Date: August 28, 2019

\begin{abstract}
Background: Among different races, the intra parotideal course of the facial nerve is very variable. Familiarity with the buccal branch pattern types have a paramount importance for meticulous manipulation and avoidance of nerve injury. This study aimed to describe the pattern of the buccal branch of facial nerve among a sample of Sudanese subjects.

Methodology: A total of 90 facial nerves were dissected, [40 cadavers (bilateral) and 10 patients (unilateral)]. Out of these 50 cases, 41 were males ( 37 cadavers and 4 patients) and 9 were females ( 3 cadavers \& 6 patients). Forty-six (51\%) were left and 44 (49\%) were right facial nerves. Photographs of intraparotideal distributions of each buccal branch were taken.

Results: The pattern of the buccal branch of the facial nerve was found as follows twenty-nine cases (32.2\%) were Type I, 14 cases were right and 15 were left. The most prevalent pattern was Type II, 39 cases (43.3\%), 23 of the cases were right and 16 cases were left. According to gender, Type I and II were the most prevalent types among males in 36 cases and 25 cases respectively. Type IV was the most common types among females accounting for 5 cases. There was similarity between both sides in relation to the most common pattern of buccal nerve branch.
\end{abstract}

Conclusion: Its fundamental to the oral and maxillofacial surgeons to have a wide exposure and detailed anatomical knowledge regarding the pattern topography of the buccal branch of the facial nerve and its relation to the parotid duct, as the facial nerve branches injury could lead to annoying poor self-image and frustrating difficult interaction.

\section{Introduction}

It is well known in the vast majority of the literature; the buccal branch can be single or double in number. It has a close relationship to the parotid duct, and it was divided to superficial and deep branches, which can be further divided to upper and lower branches as they cross the face horizontally, they predominantly supply a clinically significant part of the face [1]. For successful surgery for parotid field area, it is extremely essential to have a tremendous knowledge of the topography of the buccal branch and its relation to the parotid duct. It's worthwhile to mention that, careful selection of the surgical technique and suitable incision is highly relevant in these sorts of surgeries in relation to the parotid duct as it is essential in elucidation the pattern of the buccal nerve branch of the facial nerve [1]. A study on the relationship of the buccal branch of the facial nerve to the parotid duct was carried by Pogrel MA et al. [2], in California. In 85\% of the cadavers there was a single buccal branch of the facial nerve, whereas $15 \%$ had two branches. In $75 \%$ of cases, the nerve was inferior to the duct as it emerged from the parotid gland, whereas in $25 \%$ of cases the nerve crossed the duct, usually from superior to inferior [2].

In 2006 Saylam et al. [3], studied the anatomic landmarks of the buccal branches of the facial nerve among a sample of Turkish subjects. Thirty cadaver heads 60 specimens were dissected. The vertical and horizontal relationships between the buccal branches of the facial nerve and tragus, and parotid duct were recorded and analyzed. The buccal branches of the facial nerve were classified into four types:

Type I: A single buccal branch of the facial nerve at the point of emergence from the parotid gland and inferior to the parotid duct (Figure 1). 


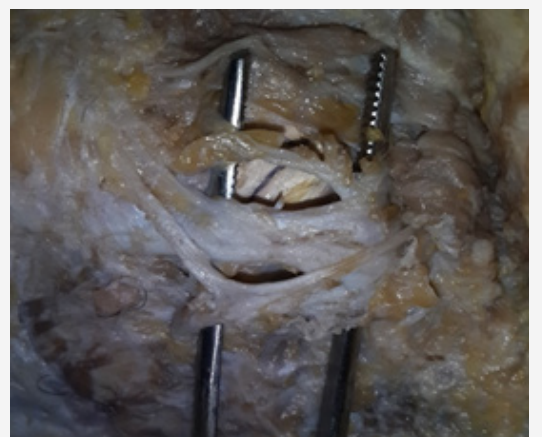

Figure 1: Type I: A Single Buccal Branch of the Facial Nerve Inferior to the Parotid Duct.

Type II: A single buccal branch of the facial nerve at the point of emergence from the parotid gland and superior to the parotid duct (Figure 2).

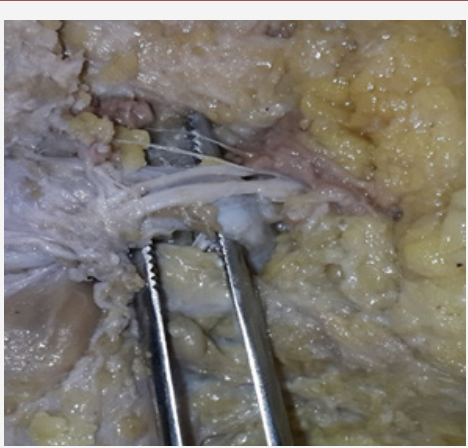

Figure 2: Type II: A Single Buccal branch of the Facial Nerve Superior to the Parotid Duct.

Type III: Buccal and other branches of the facial nerve formed a plexus (Figure 3).

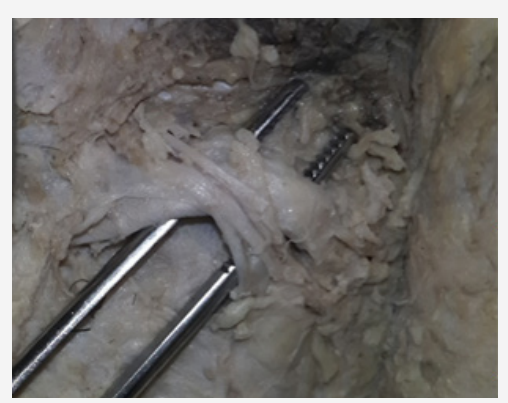

Figure 3: Type III: Buccal and other branches of the Facial Nerve formed A Plexus.

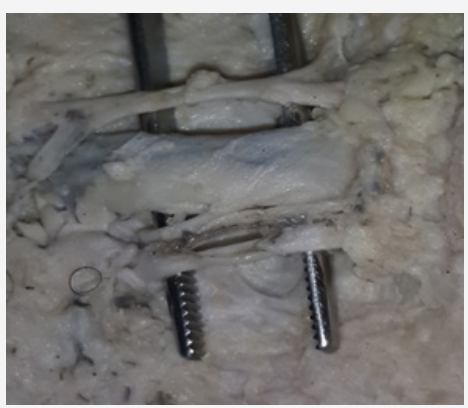

Figure 4: Type IV: Two branches of Buccal branch; one Superior and one Inferior to the Duct.
Type IV: Two branches of buccal branch; one superior and one inferior to the duct at the point of emergence from the parotid gland (Figure 4).

The investigators found the percentages of occurrence of the types as followed: type I and II were $52 \%$ and $35 \%$ respectively, the rest $13 \%$ for both type III and IV [3].

\section{Methodology}

A total of 90 facial nerves were dissected, [ 40 cadavers (bilateral) and 10 patients (unilateral)]. Out of these 50 cases, 41 were males (37 cadavers and 4 patients) and 9 were females ( 3 cadavers \& 6 patients). Forty-six (51\%) were left and 44 (49\%) were right facial nerves. The study was conducted in Khartoum Teaching Dental Hospital and the Dissection Rooms at the Department of Anatomy, medical campuses of different Universities and Bashair mortuary in Khartoum state, Sudan. Ethical approval was obtained from the University of Khartoum Faculty of Dentistry, Ethical Committee Review Board, research unit at Khartoum Teaching Dental Hospital and a signed written consent was obtained from the patients. Previous damaged intraparotid facial nerve in cadaveric dissection, patients with history of previous surgical operations in the parotid region and subjects with history of maxillofacial trauma which damaged the facial nerve were excluded from the study. Data were entered in a computer master sheet using SPSS version 16. All statistical analysis was set at 95\% confidence level, 0.2 the width of the confidence interval and the level of significance alpha 0.05 .

Pattern of the buccal branch was classified according to Saylam classification. In all cases an incision was made anterior to the tragus of the ear and extending down below the lobule of the ear. An inverted S-shaped incision was extended behind the ear and inferio-posterior to the angle of the mandible. In case of anterior parotid lesion, the incision was extended further down the neck. After elevating the flaps, the anterior border of sternocleidomastoid muscle was dissected to the mastoid process. The posterior belly of digastric muscle was visualized. The cartilaginous portion of external auditory canal was dissected to the bony portion. So, in an orthograde approach the facial nerve was identified using the two standard anatomic landmarks (the tragal pointer and the posterior belly of digastric muscle). The facial nerve was visualized and dissected peripherally, and tracing of the buccal branch was carried out. For the retrograde approach the buccal branch was used as a guide to reach the main trunk. Following dissection, schematic illustrations and photographs of the buccal branch in relation to the parotid duct were done and according to the branching pattern they were classified into groups as described by Saylam.

\section{Results}

\section{According to the side of the dissection, types of pattern of the buccal branch were identified as follows}

Twenty-nine cases (32.2\%) were Type I, 14 cases were right and 15 were left. The most prevalent pattern was Type II, 39 cases (43.3\%), 23 of the cases were right and 16 cases were left. Type III was found only in one case. Type IV was identified in 21 cases 
$(23.3 \%)$, seven of the cases were right and 14 cases were left (Table 1 \& Figure 5).

Table 1: Pattern of distribution of the buccal branch according to the side of dissection.

\begin{tabular}{|c|c|c|c|c|}
\hline \multirow{2}{*}{ Pattern of Buccal Branch } & \multicolumn{2}{|c|}{ Side of Dissection } & \multirow{2}{*}{ Total } \\
\cline { 3 - 4 } & Left & Right & \\
\hline Type I & Frequency & 15 & 14 & 29 \\
\hline & Percent & $51.72 \%$ & $48.28 \%$ & $100 \%$ \\
\hline Type II & Frequency & 16 & 23 & 39 \\
\hline & Percent & $41.03 \%$ & $58.97 \%$ & $100 \%$ \\
\hline Type III & Frequency & 1 & 0 & 1 \\
\hline & Percent & $100 \%$ & $0 \%$ & $100 \%$ \\
\hline Type IV & Frequency & 14 & 7 & 21 \\
\hline & Percent & $66.67 \%$ & $33.33 \%$ & $100 \%$ \\
\hline
\end{tabular}

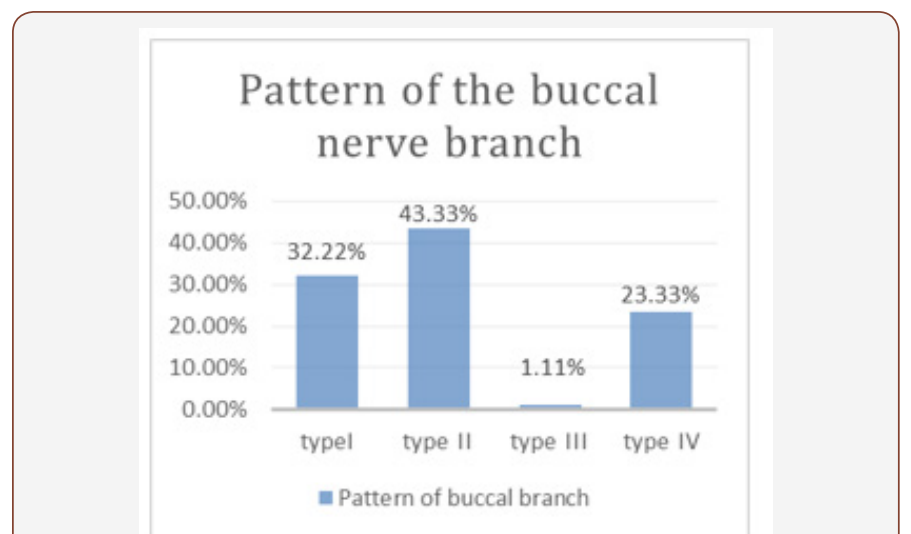

Figure 5: Distribution of the pattern of the Buccal Nerve branch.

\section{According to gender, the pattern of buccal branch was found as follows}

Type I and II were the most prevalent types among males in 36 cases and 25 cases respectively. Type IV and type I were the most common types among females accounting for 5 and 4 cases respectively (Figure 6).

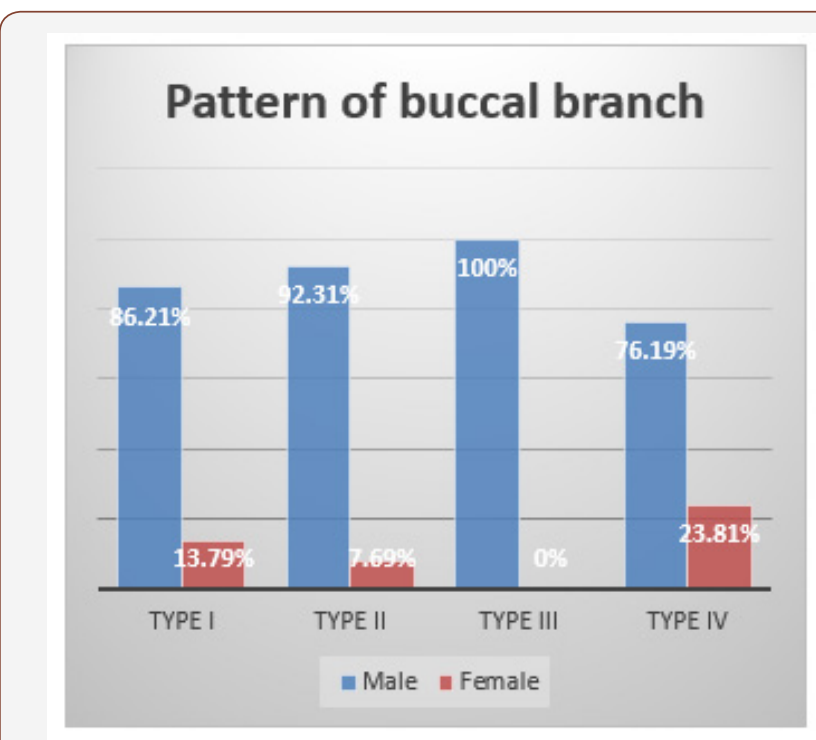

Figure 6: Distribution of Pattern of the Buccal branch according to the gender.

\section{Bilateral (40 cadaveric cases) buccal nerve branch pattern configuration was identified as follows}

Different on the two sides of dissection in 17 cases and was similar in 23 cases (Figure 7).

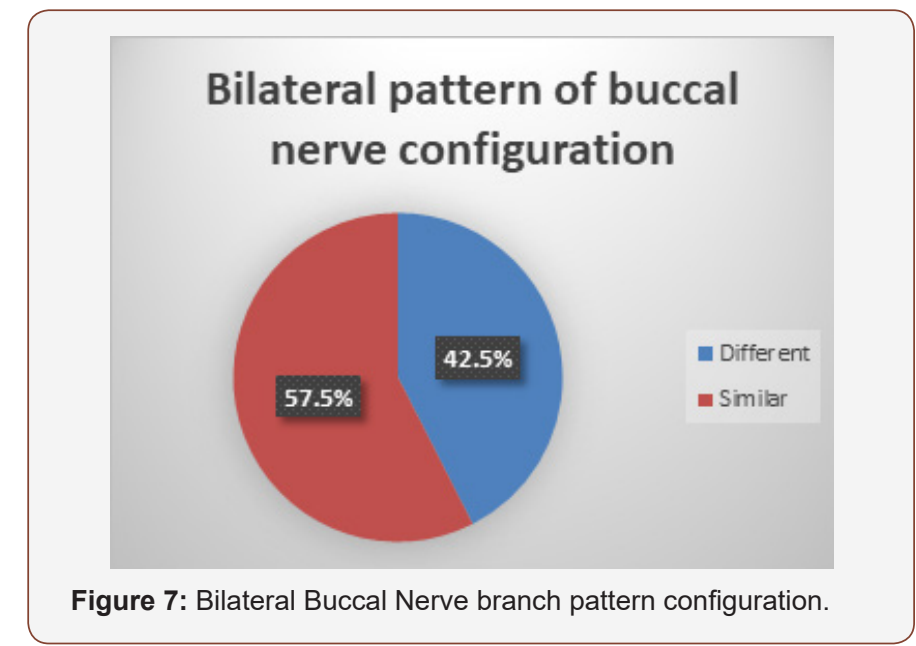

\section{Discussion}

A precise identification, localization and preservation of the facial nerve branches must precede the excision of an involved gland by proper exposure and handling that attains by means of meticulous careful dissection unless the nerve branches are to be sacrificed because of invasion by a tumor [4]. The area of greatest vulnerability for the buccal nerve is the area between the anterior border of the parotid gland before it innervates the lip elevators and a line drawn from the lateral canthus to the oral commissure anterior. Patients with facial paralysis, may experience tremendous psychosocial distress and stigma about their condition [5]. The pattern of the buccal branches of the facial nerve was classified by Saylam et al. [3] into four types according to the vertical and horizontal relationships between the buccal nerve branch and the parotid duct. Few studies were performed to record and analyse the pattern of the buccal nerve branch in relation to the parotid duct and most of them on Turkish specimens. According to Saylam classification: Type I where a single buccal nerve branch inferior to the parotid duct. Type II where a single buccal nerve branch superior to the parotid duct.

In the present study the most common pattern of the buccal nerve branch in relation to the parotid duct was type II (43.3\%) followed by type I $(32.2 \%)$. These findings were different from studies carried by Saylam et al. [3], who reported that type I was the most common pattern of buccal branch accounting for (52\%), followed by type II (35\%) respectively. So, the current study highlights a major variation in this pattern topography in relation to the saylam study, which is the main reference study, this may be due to the difference in sample size and anatomogeographical variations. Type III where the buccal nerve branch and other branches of the facial nerve formed a plexus was found in one cadaveric case in the left side of the face, which is an important surgical point as its difficult to approach this complex type during surgical dissection. 
Type IV where two branches of buccal branch; one superior and one inferior to the duct at the point of emergence from the parotid gland was found in $23.3 \%$ of the present study. Saylam et al. [3] reported that $13 \%$ of the cases where found to have mixed patterns of the buccal nerve branch. There were no reported studies in the literature that recorded and analyzed the gender distribution of the pattern of the buccal branch. The present study revealed that type I and II were the most prevalent types among males accounting for 36 and 25 cases respectively. Type IV and type I were the most common types among females accounting for 5 and 4 cases respectively.

Also, there were no reported studies in the literature on the distribution of the pattern of the buccal branch according to the side of dissection. The present study showed that type II was the most common pattern on the right and left sides of face $(58.9 \%$ and $41 \%$ ) respectively. Moreover, bilateral buccal nerve branch pattern configuration (40 cadaveric cases) of the present study was found different on the two sides of the face in $42.5 \%$ and similar in $57.5 \%$ of the specimens. In the literature there were no previous reports related to the bilateral buccal nerve branch pattern configuration.

\section{Conclusion}

It is extremely crucial for the surgeons to have familiarity and basic knowledge with the different pattern types of buccal branch of the facial nerve in order to avoid injury of it and the parotid duct during surgery, avoid the functional loss of the acting muscles which supply and enhance the quality of life. Another surgical benefit. it could describe the way of selection of parotid surgical approach specifically the retrograde one which depends commonly on the buccal branch taking the parotid duct as essential landmark. The study recommended further large cadaveric study on this field using the micro-loups which will be a major forward leap to have a challenge of detection of any tiny nerve twigs and reporting of a new pattern variation.

\section{Acknowledgement}

None.

\section{Conflict of Interest}

No conflict of interest.

\section{References}

1. Michael Monteiro ((2008)) Face and Scalp. In: Standring S (edt.), Gray's Anatomy ( $40^{\text {th }}$ edn.), The Anatomical basis of clinical practice. Elsevier publication, Netherlands, pp: 494-495.

2. Pogrel MA, Schmidt B, Ammar A (1996) The relationship of the buccal branch of the facial nerve to the parotid duct. J Oral Maxillofac Surg 54(1): 71-73.

3. Saylam C, Ucerler H, Orhan M, Ozek C (2006) Anatomic landmarks of the buccal branches of the facial nerve. Surg Radiol Anat 28: 462-467.

4. Salasche S, Bernstein G, Senkarik M (1988) Surgical anatomy of the skin. Norwalk (CT): Appleton and Lange p: 8-10.

5. Andreas Kehrer, Simon Engelmann, Marc Ruewe, Sebastian Geis, Taeger C, et al. (2019) Anatomical Study of the Zygomatic and Buccal Branches of the Facial Nerve: Application to facial reanimations procedures. Clin Anat 23: 1-9. 